\title{
Acute Kidney Injury and Progressive Diabetic Kidney Disease: An Epidemiological Perspective
}

This article was published in the following Dove Press journal:

International Journal of Nephrology and Renovascular Disease

\author{
Ravindra Attur Prabhu (D) \\ Srinivas V Shenoy (D)' \\ Shankar Prasad Nagaraju (iD) \\ Dharshan Rangaswamy (iD) \\ Indu Ramachandra Rao (D) \\ Mohan V Bhojaraja (iD) \\ Deepak Nayak $M$ iD $^{2}$ \\ Sindhura Lakshmi Koulmane \\ Laxminarayana (iD) ${ }^{2}$ \\ Karan Saraf ${ }^{1,3}$ \\ Ashok Ramaswamy ${ }^{1,4}$ \\ 'Department of Nephrology, Kasturba \\ Medical College Manipal, Manipal \\ Academy of Higher Education, Manipal, \\ Karnataka, India; ${ }^{2}$ Department of \\ Pathology, Kasturba Medical College \\ Manipal, Manipal Academy of Higher \\ Education, Manipal, Karnataka, India; \\ ${ }^{3}$ Department of Nephrology, Excelcare \\ Hospitals, Guwahati, Assam, India; \\ ${ }^{4}$ Department of Nephrology, Saveetha \\ Medical College, Chennai, Tamil Nadu, \\ India
}

Purpose: Diabetic kidney disease (DKD) represents a unique subset of patients with chronic kidney disease (CKD). Acute kidney injury (AKI) is implicated in DKD progression; however, their interplay is not studied well. We studied risk factors for AKI and the effect of AKI on disease progression in a homogeneous group of patients with DKD.

Patients and Methods: We conducted a retrospective open cohort study of patients with DKD at a single tertiary care centre between August 2016 - August 2019. Patients with a minimum follow-up of 2 years were included in the study. The incidence, etiology and risk factors for AKI were studied. The primary outcome studied was the effect of AKI on reduction in estimated glomerular filtration rate (eGFR) in DKD. Loss in eGFR by 50\% and need for renal replacement therapy or reaching CKD stage V were studied as secondary outcomes.

Results: Two hundred and ninety-two DKD patients meeting the study criteria with a follow-up of $29.57( \pm 4.3)$ months were included. The incidence of AKI was $31.1 \%$. Sepsis was the most common etiology (61\%). Proteinuria was an independent risk factor for AKI after adjusting for covariates (adjusted OR - 1.158; 95\% CI $(1.018-1.316) ; \mathrm{p}=0.025)$. In patients with AKI, median decline in eGFR was $10.29 \mathrm{~mL} / \mathrm{min} / 1.73 \mathrm{~m}^{2} /$ year (IQR-5.5813.84) which was significantly higher compared to patients with no AKI [eGFR 7.25 (IQR 5.06-11.38); p-0.014]. On subgroup analysis, sepsis-induced AKI (versus non-sepsis AKI; $\mathrm{p}<0.001$ ) and higher AKI stage (stage $2 / 3$ versus stage $1 ; \mathrm{p}=0.019$ ) were associated with a faster decline in eGFR.

Conclusion: AKI is common in patients with DKD with sepsis being the most common etiology. AKI in diabetic kidney disease is associated with a faster decline in eGFR. Baseline proteinuria is an independent risk factor for AKI.

Keywords: diabetic kidney disease, acute kidney injury, GFR decline, proteinuria, sepsis

\section{Introduction}

Diabetic kidney disease (DKD) is a well-established and major complication of diabetes mellitus (DM). An estimated $20 \%$ to $40 \%$ of patients develop DKD in their lifetime. ${ }^{1,2}$ It is often punctuated by an unpredictable course, heralded by a nonlinear fall in glomerular filtration rate (GFR) compared to other etiologies of chronic kidney disease (CKD). ${ }^{3}$ Risk factors for CKD progression, in general, include various modifiable and non-modifiable factors like gender, racial differences, genetics, presence of co-existing comorbid illness, metabolic factors and acute kidney injury (AKI). ${ }^{4}$ AKI and CKD are now considered as an integrated clinical syndrome in nephrology practice. ${ }^{5}$ Long-term consequences of AKI include progression to end-stage kidney disease (ESKD), adverse cardiovascular and
Correspondence: Srinivas V Shenoy Department of Nephrology, Kasturba Medical College Manipal, Manipal Academy of Higher Education, Manipal, Karnataka, India

Tel +9l 9844374705

Email shenoy.srinivas@manipal.edu

International Journal of Nephrology and Renovascular Disease 202|:|4 23-3| 
cerebrovascular events together resulting in increased morbidity, decreased quality of life and increased mortality. ${ }^{6}$ Studies have shown AKI not only as a risk factor for progressive CKD but CKD itself being a risk factor for recurrent $\mathrm{AKI}^{7}{ }^{7}$ However despite this close association between $\mathrm{AKI}$ and $\mathrm{CKD}$, their interplay is often complex and poorly understood.

DKD is one of the most common causes of ESKD worldwide. $^{8}$ Type $2 \mathrm{DM}$ with or without underlying CKD has been independently implicated as a risk factor for AKI. ${ }^{9}$ Hence, the impact of AKI on patients with DKD assumes widespread importance. The relationship between $\mathrm{CKD}$ and $\mathrm{AKI}$ has been explored in the previous literature which included either only elderly patients, ${ }^{10}$ hospitalized patients, ${ }^{11}$ postoperative patients ${ }^{12,13}$ which were heterogeneous study populations (including non-CKD and CKD with different etiologies). There are very few studies that have looked into the relationship between AKI and diabetes or AKI in diabetic kidney disease. ${ }^{14-16}$ Though AKI is described as a risk factor for DKD progression, intricate variables such as incidence, etiology, risk factors, impact on the rate of decline in GFR have not been well studied.

Our study was intended to look into the relationship between AKI in a homogeneous group of patients with DKD from an epidemiological perspective. Here we studied the incidence, etiology and risk factors for AKI and the effect of AKI on reduction in estimated glomerular filtration rate (eGFR) in DKD. We also looked into how different stages and different etiologies of AKI may affect the renal outcomes. This study could potentially help in better understanding the AKI characteristics in DKD, which would then facilitate both to identify "at risk" patients who may be prone to AKI and to chart the progression of their renal disease.

\section{Patients and Methods}

The study was a retrospective open cohort design. All procedures followed were in accordance with the ethical standards of the responsible committee on human experimentation (institutional and national) and with the Helsinki Declaration of 1975, as revised in 2008. All patients visiting the outpatient departments or getting admitted at Kasturba Medical College and Hospital, Manipal between August 2016 - August 2019, fulfilling the study criteria, with a minimum follow-up of 2 years were included in the study. After successfully seeking an approval from the institutional ethics committee (Registration No. ECR/146/Inst/KA/2013) (IEC 49/2016), the laboratory data of the patients were retrieved from the electronic hospital management system using the international classification of diseases 10 (ICD-10) coding system. The corresponding clinical data were obtained from case files that were available in the medical records section. Patient data confidentiality was maintained. Our institutional ethics committee approved a waiver of consent as this was a retrospective study. Only the primary investigator had access to the medical records. There were no direct identifiers collected for the patient apart from hospital numbers which were later coded to patient number $1,2,3 \ldots$ to maintain confidentiality during data analysis and publication.

\section{Inclusion Criteria}

- 1) Type 2 DM patients aged $>18$ years with CKDstage G1-G4 as per the Kidney Disease: Improving Global Outcomes (KDIGO) guidelines ${ }^{17}$ and with diabetic nephropathy based on the following:

- Presence of persistent proteinuria with urine protein creatinine ratio $>300 \mathrm{mg} / \mathrm{g}$ creatinine, with additional evidence of diabetic retinopathy and absence of clinical or laboratory evidence of any other renal disease18 OR patients with a renal biopsy done showing features of diabetic nephropathy in the absence of diabetic retinopathy.

\section{Exclusion Criteria}

1. Patients with Type 2 Diabetes Mellitus with a nondiabetic kidney disease

2. Patients with CKD stage 5 (eGFR $<15 \mathrm{~mL} / \mathrm{min}$ ).

Baseline clinical characteristics like age, body mass index (BMI), gender, presence of cardiovascular disease (left ventricular dysfunction (systolic/diastolic), prior acute coronary syndrome or prior cerebrovascular accident), use of angiotensin-converting enzyme inhibitors/angiotensin receptor blockers (ACEi/ARB) were collected. eGFR was calculated as per the CKD Epidemiology collaboration formula (CKD EPI). ${ }^{19}$ Patients were grouped into AKI/ No AKI and their baseline characteristics and outcomes were compared. In cases of multiple outpatient visits or hospital admissions, we considered the visit during which AKI was diagnosed. Peak creatinine value during the acute episode was used to calculate the AKI staging and eGFR. The last creatinine value obtained within the study period and at least 3 months following the AKI episode were taken to calculate the eGFR decline for these patients. 
For patients who did not develop AKI during follow-up visits, we took the baseline creatinine during the screening and the last creatinine value obtained within the study period to calculate the eGFR decline.

\section{Definitions}

1. AKI and its staging: Episodes of acute kidney injury (AKI) were documented and staged using serum creatinine criteria of KDIGO guidelines. $^{20}$ Urine output criteria were not used. AKI was defined as an increase in serum creatinine by $0.3 \mathrm{mg} / \mathrm{dl}$ within 48 hours or $>1.5$ times known baseline value occurring within 1 week. Staging of AKI was done as below

Stage 1: $1.5-1.9$ times above baseline OR $>0.3 \mathrm{mg} / \mathrm{dl}$ increase.

Stage 2: Increase by 2-2.9 times baseline

Stage 3: Increase by $>3$ times baseline or increase in serum creatinine to $>4 \mathrm{mg} / \mathrm{dl}$ or the initiation of renal replacement therapy

2. Sepsis: Sepsis was defined as per the 3rd International consensus definition released in 2016. ${ }^{21}$ A combination of evidence of infection and 2 out of 3 criteria in quick Sequential Organ Failure Assessment (qSOFA) score was used.

\section{Study Outcomes}

The primary outcome was a measure of the decline in eGFR $\mathrm{mL} / \mathrm{min} / 1.73 \mathrm{~m}^{2} /$ year. Secondary outcomes that were assessed included eGFR loss by $50 \%$ or attainment of Stage $5 \mathrm{CKD} / \mathrm{Need}$ for renal replacement therapy (RRT) at last follow-up. Risk factors associated with the occurrence of AKI were also studied. Details of AKI etiology were also collected. Patients with sepsis-associated AKI were compared with those with other etiologies to assess the outcomes.

\section{Statistical Analysis}

Baseline characteristics were expressed in terms of mean \pm standard deviation (SD) (normal distribution), median interquartile range (IQR) (non-normal distribution) for continuous variables and as number (percentages) for categorical data. Comparisons were made using $t$-tests (normal distribution) and Mann-Whitney $U$-test (non-normal distribution) test for continuous variables. Categorical variables were compared with Chi-square or Fisher exact tests.
Risk factors were compared between AKI and Non-AKI groups with univariate analysis. Significant risk factors from univariate analysis were used to construct a model for multivariate (binary) logistic regression. Hosmer and Lemeshow goodness of fit test was used to validate the model. Multicollinearity among significant risk factors was tested by collinearity statistics using Tolerance and Variation inflation factor (VIF) values. Renal outcomes were also compared for the severity of AKI based on staging and also based on the etiology of AKI (sepsis versus other etiology). For patients with more than 1 episode of AKI, the AKI episode with higher staging was taken for analysis. A p-value of $<0.05$ was taken as significant. The statistical analysis was done by IBM SPSS Statistics for Windows, version 20 (IBM Corp., Armonk, N.Y., USA). A complete case analysis was done and cases with missing data were excluded from the analysis.

\section{Results}

\section{Baseline Characteristics}

Three hundred and twelve patients meeting the study criteria were screened into the study and subsequently, 292 patients completing a minimum of 2 years of follow-up were included in the analysis (Figure 1). Baseline characteristics are as per Table 1. The mean age was 59.03 $( \pm 10.23)$ years. Median eGFR at baseline was 52.72 (IQR- 35.52-71.09). Patients with CKD stage G3 were the highest in number 116 (39.7\%). There were 54.1\% of patients on either angiotensin-converting enzyme (ACE) inhibitors or angiotensin receptor blockers (ARB). Fortyone percent of patients had baseline cardiovascular disease.

\section{AKI Characteristics, Etiology, Risk Factors}

Overall, 91/292 (31.1\%) patients had AKI, of which 17 $(16.03 \%)$ required renal replacement therapy. Total episodes of AKI were 106 with 14 patients having $>1$ episode. One-third of patients had AKI stage 3 (Table 2) Sepsis was the commonest etiology in 65/106 episodes (61\%.) (Figure 2). Patients with AKI had a lower baseline eGFR (mL/min/1.73m²) (51.30 (IQR-37.15-70.72) versus 54.65 (IQR-39.15-69.31); p-0.042) and higher proteinuria (1.64 (IQR - 0.63-3.42) versus 1.1 (IQR 0.50-2.55); $\mathrm{p}=$ $0.021 ; \mathrm{g} / \mathrm{g}$ creatinine) (Table 1). Binary logistic regression analysis showed that urine protein creatinine ratio (UPCR) was independently associated with the occurrence of AKI- 
Adjusted Odds ratio (OR) 1.158; 95\% CI (1.018-1.316); $\mathrm{p}=0.025$

\section{AKI Outcomes}

Mean follow-up of the patients was $29.57( \pm 4.3)$ months.

Overall the median decline in eGFR was $7.65 \mathrm{~mL} / \mathrm{min} /$ $1.73 \mathrm{~m}^{2} /$ year (IQR- 5.20-13.02) with 95 (32.5\%) patients having an eGFR loss of $50 \%$ or more and $64(21.9 \%)$ reaching stage $5 \mathrm{CKD} /$ need for RRT (Table 3).

Patients with AKI had a significant decline in eGFR/ year (10.29 (IQR-5.58-13.84)) when compared with patients without AKI (7.25 (IQR 5.06-11.38); p-0.014)

AKI was a significant association for eGFR loss of 50\% (Odds Ratio 2.56; 95\% CI (1.528-4.312); $<<0.001$ ) (Table 3)

\section{Subgroup Analysis - AKI Staging and Etiology}

Patients with stage 2 or 3 AKI had a faster decline in eGFR ( $\mathrm{mL} / \mathrm{min} / 1.73 \mathrm{~m}^{2} /$ year) compared to stage 1 AKI [11.05 (IQR 6.66-15.24) versus 7.34 (IQR 4.65-11.54); $\mathrm{p}=0.019$ ] (Table 4). There were 17 patients with AKI episodes requiring hemodialysis. Patients who required hemodialysis had a median decline in eGFR $(\mathrm{mL} / \mathrm{min} /$ $1.73 \mathrm{~m}^{2} /$ year) of 13.18 (IQR 7.07-16.45) versus 9.37 (IQR 5.49-13.54) for patients with AKI but who did not require hemodialysis $(\mathrm{p}=0.058)$. In the subgroup of patients requiring hemodialysis, $58.8 \%$ (10 out of 17) reached an end point of eGFR loss of 50\% and $41.2 \%$ (7 out of 17) reached an end point of Stage 5 CKD at last follow-up.

Sepsis-associated AKI episodes resulted in a faster decline in eGFR $\left(\mathrm{mL} / \mathrm{min} / 1.73 \mathrm{~m}^{2} /\right.$ year) $[11.75$ (IQR 7.50-15.09) versus 6.89 (IQR 4.65-10.65); p-value 0.001)] (Table 5)

\section{Discussion}

The prevalence of CKD is pegged to be nearly one-third in patients with DM. ${ }^{1,22}$ Patients with diabetic kidney disease are known to develop episodes of AKI where either there is no recovery in the short term or may result in accelerated progression of kidney disease in the long term. ${ }^{23}$ Additionally, DM is a risk factor for AKI irrespective of underlying CKD status ${ }^{24}$. Evidence for overall risk for AKI in a community-based cohort was obtained from a retrospective analysis of the United Kingdom (UK) general practice database by Girman et al in which they concluded that Type $2 \mathrm{DM}$ was a significant risk factor for AKI. ${ }^{14}$ A recent observational study by Hapca et al concluded from their cohort of patients that in the absence and presence of CKD, the rates of AKI were nearly 5 times and 2 times, respectively, for patients with diabetes compared to the control groups. ${ }^{16}$ Mechanisms postulated to explain the susceptibility of DM to AKI include endothelial dysfunction due to upregulation of vasoconstrictor endothelin -1 (ET-1), downregulation of nitric oxide production, ${ }^{25,26}$ proximal tubular injury secondary to upregulation of inflammatory cytokines like tumour necrosis factor (TNF)- $\alpha$, interleukin (IL)-1, IL-6, maladaptive repair process which causes scarring and tubulointerstitial fibrosis leading to CKD progression and ESKD. $^{27,28}$

We studied the incidence of AKI in our cohort as most of the observational studies included incidences of AKI only in hospitalized patients, critically ill, ${ }^{11}$ elderly, ${ }^{29}$ postsurgical patients ${ }^{30}$. The calculated yearly incidence of AKI in our cohort was $12.6 \%$ (31.1\% patients over a mean follow up of $29.57( \pm 4.3)$ months). This is a significant departure from published studies by Thakar et $\mathrm{al}^{31}$ and Monseu et $\mathrm{al}^{32}$ who reported a yearly incidence of AKI of $2.8 \%$ and $5.2 \%$, respectively. ${ }^{31,32}$ AKI episodes were more severe in our cohort with nearly a third of episodes (35/ 106) being classified as stage 3 compared to $12 \%^{31}$ and $6 \%{ }^{32}$ in other studies. It is important to note that while in our study we dealt with patients with established DKD, the cohort of patients in the comparative studies had patients with only diabetes and with few having DKD. The baseline eGFR in our patients was $52.72 \mathrm{~mL} / \mathrm{min} / 1.73 \mathrm{~m}^{2}$ (IQR- 35.52-73.09) whereas it was $81.1( \pm 25.9) \mathrm{mL} / \mathrm{min} /$ $1.73 \mathrm{~m}^{2}$ and $73.6( \pm 23.9) \mathrm{mL} / \mathrm{min} / 1.73 \mathrm{~m}^{2}$ in studies by Thakar et al and Monseu et al, respectively. In the study by Sykes et al in the salford kidney study (SKS) cohort, where patients with CKD of different etiologies were followed up, $16.4 \%$ had DKD. The overall incidence of AKI in that cohort was a reported $28.11 \%$ (643 out of 2287), with a yearly incidence of $11 \%$. The baseline eGFR in this cohort was $28.4 \mathrm{~mL} / \mathrm{min} / 1.73 \mathrm{~m}^{2.33}$ The variations in the incidence and severity of AKI episodes, between our and the other studies can be explained by differences in baseline renal functions of patients enrolled in the study. A lower baseline eGFR could have adversely increased the susceptibility of developing an AKI episode, with a corresponding increment in the AKI stage. A similar incidence of AKI in the salford kidney study cohort supports our hypothesis. 
312 patients with diabetes visiting OPD or admitted screened for inclusion and exclusion criteria August 2016- August 2017
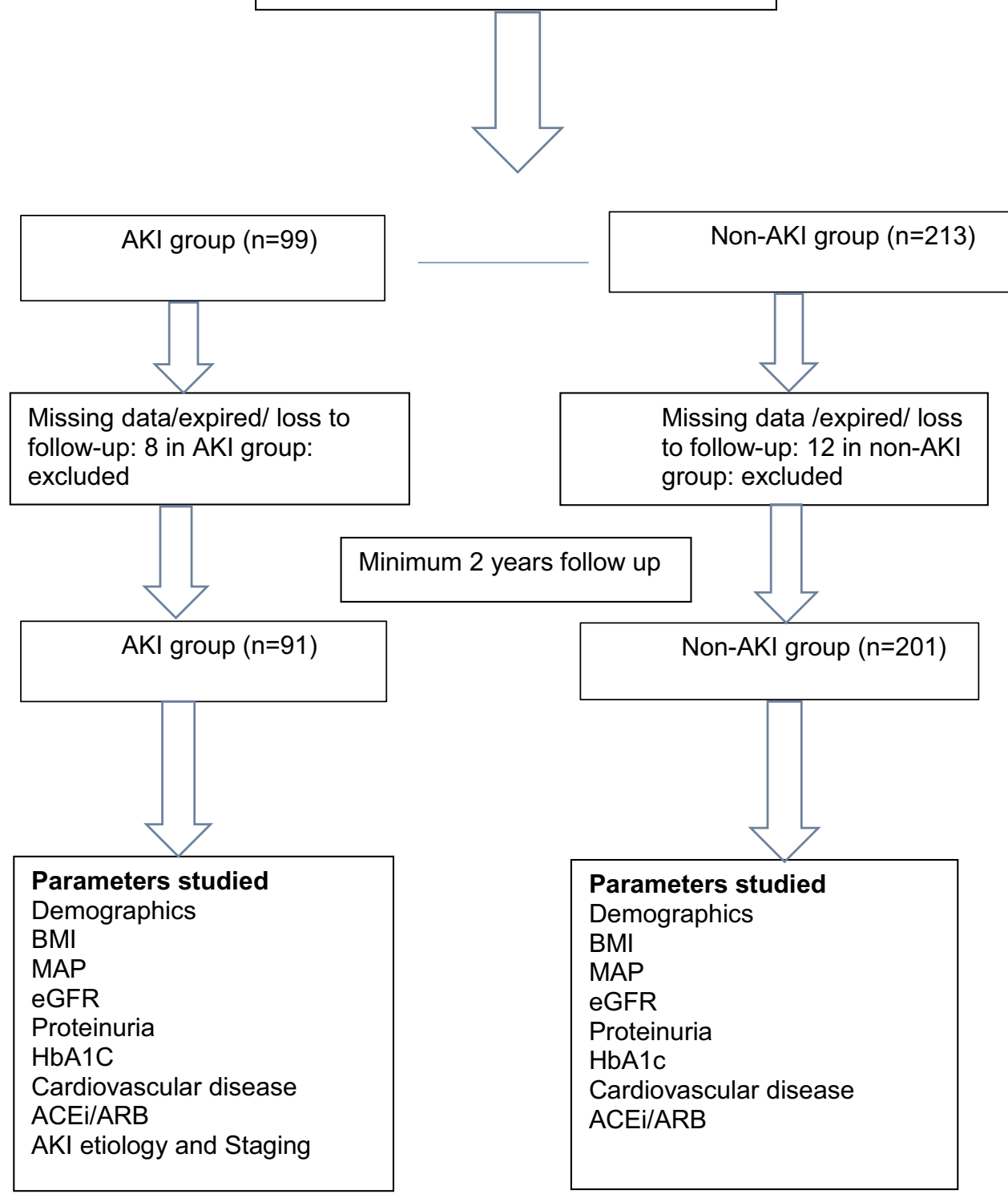

Figure I Flow chart of participants.

Abbreviations: OPD, outpatient department; AKI, acute kidney injury; BMI, body mass index; MAP, mean arterial pressure; ACEi/ARB, angiotensin-converting enzyme inhibitors/angiotensin receptor blockers; HbAIc, glycosylated haemoglobin; eGFR, estimated glomerular filtration rate.

In our study, patients with AKI had a lower baseline eGFR and a higher degree of proteinuria on univariate analysis and proteinuria was found to be independently associated with AKI incidence on logistic regression. For every $1 \mathrm{~g} / \mathrm{g}$ increase in proteinuria (UPCR) there was a $15.8 \%$ increase in the risk of AKI. The study by Monseu et al also concluded that a lower eGFR and a higher albuminuria were significantly associated with $\mathrm{AKI}^{32}$. In a large cohort study in Alberta, Canada, among hospitalized patients with AKI, moderate to heavy proteinuria was a risk factor for AKI across all eGFR groups. ${ }^{34}$

We further noted that patients with AKI had a significantly faster rate of decline in eGFR. In addition, significantly more patients with AKI reached an end point of 50\% eGFR reduction when compared to those without AKI. The results are in concordance with other studies done in CKD patients with 
Table I Baseline Characteristics of Patients

\begin{tabular}{|c|c|c|c|c|}
\hline Parameters & Overall $(n=292)$ & AKI (n=9I) & No AKI $(n=20 I)$ & p-value \\
\hline Age (years) & $59.03( \pm 10.23)$ & $58.53( \pm 9.79)$ & $59.91( \pm 10.57)$ & 0.900 \\
\hline Males (n)(\%) & 203(69.5) & $69(75.8)$ & $134(66.6)$ & 0.114 \\
\hline BMI $\left(\mathrm{kg} / \mathrm{m}^{2}\right)$ & 25.11 (IQR-22.92-26.82) & 25.0 I(IQR-22.89-27.53) & 24.52 (IQR-22.48-26.29) & 0.502 \\
\hline Mean arterial pressure $(\mathrm{mm}$ of $\mathrm{Hg})$ & $103.11( \pm 10.97)$ & $102.56( \pm 11.09)$ & $103.56( \pm 10.93)$ & 0.565 \\
\hline Use of ACEi/ARBs & $158(54.1)$ & $48(54.7)$ & $110(52.7)$ & 0.753 \\
\hline HbAIC \% & 8.25 (IQR 7.04-9.97) & 8.41 (IQR - 7.35-10.02) & 8.1 (IQR-7.00-10.30) & 0.694 \\
\hline Cardiovascular disease (n)(\%) & $120(4 \mid .1)$ & $38(41.7)$ & $82(40.7)$ & 0.872 \\
\hline Urine protein creatinine ratio (g/g) & I.2 (IQR- 0.5I-2.72) & $1.64(\mathrm{IQR}-0.63-3.42)$ & I.I (IQR 0.50-2.55) & $0.021 *$ \\
\hline eGFR $\left(\mathrm{mL} / \mathrm{min} / 1.73 \mathrm{~m}^{2}\right)$ & 52.72 (IQR- 35.52-7I.09) & 5I.30.(IQR-37.15-70.72) & 54.65 (IQR-39.I5-69.3I) & $0.042^{*}$ \\
\hline Serum creatinine $(\mathrm{mg} / \mathrm{dl})$ & I.4 (IQR I.00-I.80) & I.4 (IQR I.00-I.85) & I.3 (IQR I.I0-I.70) & 0.611 \\
\hline \multicolumn{4}{|l|}{ CKD staging (n)(\%) } & \multirow[t]{5}{*}{$0.28 I$} \\
\hline Stage G I & $39(13.4)$ & $12(13.2)$ & $27(13.4)$ & \\
\hline Stage G2 & $80(27.4)$ & $29(31.8)$ & 51 (25.4) & \\
\hline Stage G3 & 116 (39.7) & $38(4 I .8)$ & $78(38.8)$ & \\
\hline Stage G4 & $57(19.5)$ & $12(13.2)$ & $45(22.3)$ & \\
\hline
\end{tabular}

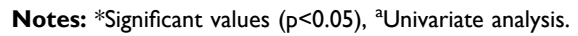

Abbreviations: BMI, body mass index; ACEi, angiotensin converting enzyme inhibitors; ARBs, angiotensin receptor blockers; HbAlc, glycosylated hemoglobin; IQR, interquartile range.

different etiologies. ${ }^{7,33,35-37}$ Case in point are the findings of both, Thakar et al and Monseu et al done in diabetic patients demonstrating that $\mathrm{AKI}$ is a risk factor for $\mathrm{CKD}$ progression in patients with low baseline GFR. ${ }^{31,32}$ AKI in an already compromised kidney function causes additional damage, tubulointerstitial fibrosis, thus accelerating the progress of GFR decline. Moreover, a compromised renal reserve makes it more vulnerable to additional insult. ${ }^{38}$

The severity of AKI is associated with a faster progression of CKD. Stage 2/3 AKI was associated with a steeper decline in eGFR as compared to patients with stage $1 \mathrm{AKI}$ in our cohort. Patients requiring dialysis had a faster decline in eGFR compared to the patients who had AKI but did not require dialysis though this association did not reach statistical significance. These findings are similar to studies by Thakar et $\mathrm{al}^{31}$ done in diabetic patients and Neyra et $\mathrm{al}^{39}$ done in CKD patients with sepsis-associated critical illness. Diabetes is also nefariously linked with an immune dysfunction; which includes defects in both, humoral and cell-mediated immunity. With an added neutrophil dysfunction, a diabetic state cumulatively leads to an increased susceptibility to sepsis. ${ }^{40,41}$ In a recent meta-analysis of sepsis-associated AKI, DM has been shown to be an independent risk factor ${ }^{42}$. That sepsis and diabetes are the most common risk factors for AKI in the critically ill has been shown in many studies ${ }^{11,43,44}$. However, studies looking into this aspect in patients with $\mathrm{DKD}$ or $\mathrm{CKD}$, in general, are lacking. Most conclusions have been derived on patients with $\mathrm{CKD}$ in a subset of the patients studied. We found sepsis to be the leading etiology for AKI in $61 \%$ of episodes. In addition, patients with a sepsis-associated AKI showed greater decline in eGFR which was in stark contrast compared to other etiologies. The prior studies ${ }^{16,31,32}$ done on patients with diabetes mellitus and diabetic kidney disease did not study the causes for AKI. We acknowledge that sometimes there could be an overlap in the causes leading

Table 2 Acute Kidney Injury - Characteristics (Total Patients n=292)

\begin{tabular}{|c|c|}
\hline Total patients with AKI - n (\%) & 9I (3I.I) \\
\hline Total patients with $>$ I AKI episode & 14 \\
\hline Total AKI episodes - n & 106 \\
\hline AKI staging - n (\%) Stage I & $34(31.9)$ \\
\hline Stage 2 & $37(35.2)$ \\
\hline Stage 3 & $35(33)$ \\
\hline Need for RRT - n(\%) & $17(16)$ \\
\hline
\end{tabular}




\section{Etiology of AKI}

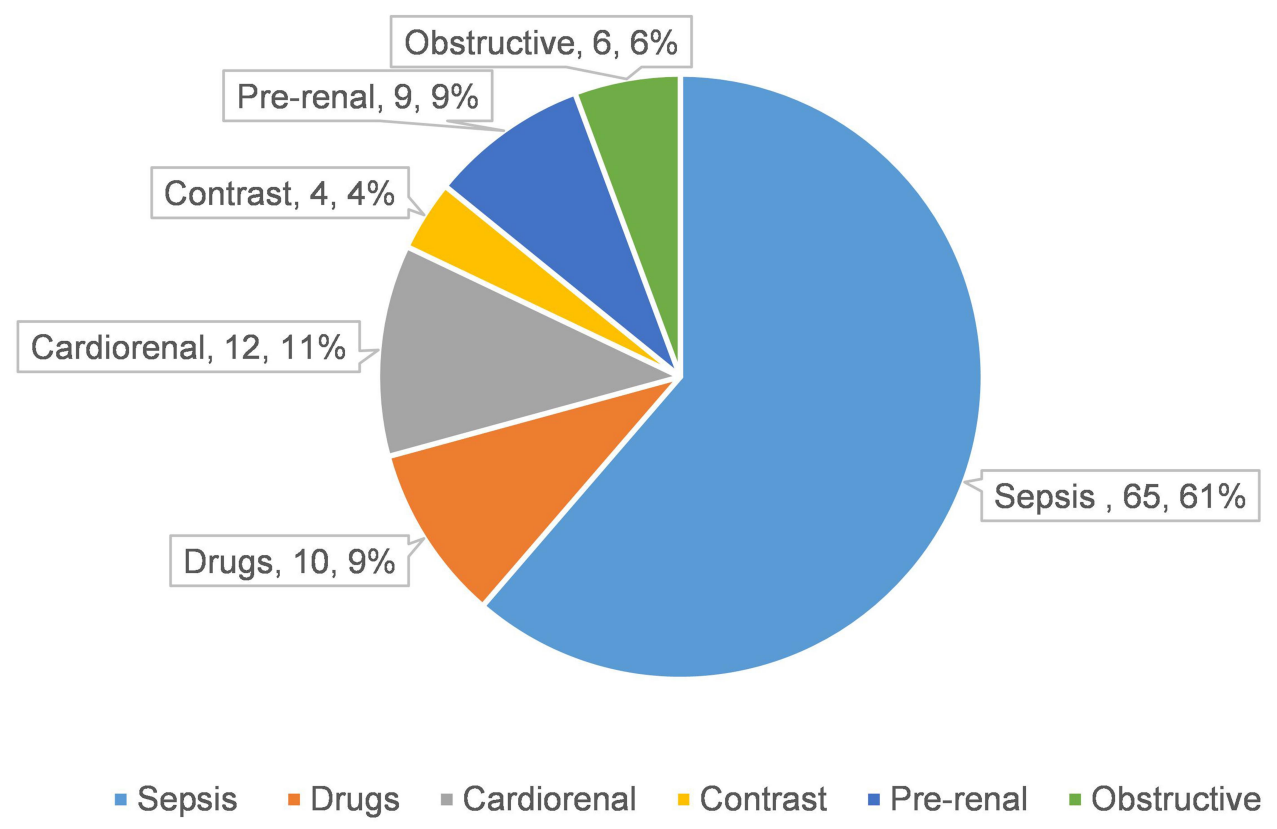

Figure 2 Etiology of AKI.

to AKI which can make it difficult to pinpoint an exact etiology. However, all patients presenting with AKI were assessed for signs of hypovolemia, they were then treated with fluid boluses if hypovolemia was confirmed. Urinary tract obstruction was ruled out by ultrasonography and all nephrotoxic drugs were stopped at admission. When AKI persisted despite these measures, we assigned the diagnosis as sepsis when they fulfilled the definition of sepsis ${ }^{21}$.

Table 3 Outcomes in AKI

\begin{tabular}{|c|c|c|c|c|}
\hline Outcome & Overall $(n=292)$ & AKI (n=9I) & No AKI (n=20I) & p-value \\
\hline eGFR decline $\left(\mathrm{mL} / \mathrm{min} / 1.73 \mathrm{~m}^{2} /\right.$ year $)$ & 7.65 (IQR 5.20-13.02) & 10.29 (IQR-5.58-13.84) & 7.25 (IQR 5.06-II.38) & $0.014^{*}$ \\
\hline eGFR loss by $50 \%(n)(\%)$ & $95(32.5)$ & $43(47.3)$ & $52(25.8)$ & $<0.001 *$ \\
\hline Stage 5 CKD/Need for RRT (n)(\%) & $64(21.9)$ & $25(27.4)$ & $39(19.4)$ & 0.123 \\
\hline
\end{tabular}

Note: *Significant P-value $(<0.05)$.

Abbreviation: RRT, renal replacement therapy.

Table 4 AKI Outcomes as per Staging

\begin{tabular}{|c|c|c|c|}
\hline Outcome & AKI Stage I $n=29$ & AKI Stage 2 and $3 n=62$ & p-value \\
\hline eGFR decline $\left(\mathrm{mL} / \mathrm{min} / 1.73 \mathrm{~m}^{2} /\right.$ year $)$ & 7.34 (IQR 4.65-II.54) & II.05 (IQR 6.66-I5.24) & $0.019 *$ \\
\hline eGFR loss by $50 \%(n)(\%)$ & $12(4 \mid .3)$ & $3 I(50)$ & 0.443 \\
\hline Stage 5 CKD/Need for RRT (n)(\%) & $8(27.6)$ & $17(27.4)$ & 0.987 \\
\hline
\end{tabular}

Note: *Significant P-value $(<0.05)$.

Table 5 AKI Outcomes - Sepsis AKI versus Non-Sepsis AKI

\begin{tabular}{|l|l|l|l|}
\hline Outcome & Sepsis AKI (n=56) & Non Sepsis AKI (n=35) & p-value \\
\hline eGFR decline $\left(\mathrm{mL} / \mathrm{min} / \mathrm{I} .73 \mathrm{~m}^{2} /\right.$ year) & $11.75(\mathrm{IQR} 7.50-15.09)$ & $6.89(\mathrm{IQR} 4.65-10.65)$ & $<0.00 I^{*}$ \\
eGFR loss by 50\% (n)(\%) & $28(50)$ & $15(42.9)$ & 0.507 \\
Stage 5 CKD/Need for RRT $(\mathrm{n})(\%)$ & $15(26.8)$ & $10(28.6)$ & 0.853 \\
\hline
\end{tabular}

Note: *Significant P-value $(<0.05)$. 
The strength of our study was that we studied a homogeneous cohort of patients with diabetic kidney disease. In the same cohort, we managed to study the incidence, risks, etiology as well as renal outcomes. Our study may have important clinical implications for practice. Firstly, patients with diabetes and kidney disease are more prone to AKI. Thus, we may need to monitor kidney functions frequently. Since ours was a retrospective cohort with irregular followups, we cannot comment on the appropriateness of the frequency. However, prospective studies may look into this aspect. Secondly, we have shown that sepsis is still a common cause for AKI for diabetic CKD patients even outside the critical care/surgical settings which should make us more vigilant to rule out any source of sepsis in a patient with AKI and underlying DKD. We acknowledge that our study does have its limitations. The design of the study, being a single-centre study with a retrospective design. We performed a complete case analysis and had to exclude cases with missing data/no follow up.

\section{Conclusions}

$\mathrm{AKI}$ is common in patients with DKD and so is sepsis as a cause for AKI. AKI, severity of AKI and presence of sepsis is associated with a faster decline in eGFR. Proteinuria is independently associated with AKI incidence. Prospective studies looking into strategies dealing with AKI prevention, prevention of sepsis are needed to develop interventions that may slow the progression of DKD.

\section{Ethics Approval}

Approval from the institutional ethics committee (Registration No. ECR/146/Inst/KA/2013) (IEC 49/2016) was obtained.

\section{Funding}

No funding was received for this work.

\section{Disclosure}

The authors report no conflicts of interest for this work.

\section{References}

1. Reutens AT. Epidemiology of diabetic kidney disease. Med Clin North Am. 2013;97(1):1-18. doi:10.1016/j.mena.2012.10.001

2. Collins AJ, Foley RN, Gilbertson DT, Chen S-C. United States renal data system public health surveillance of chronic kidney disease and end-stage renal disease. Kidney Int Suppl. 2015;5(1):2-7. doi:10.1038/ kisup. 2015.2

3. Li L, Astor BC, Lewis J, et al. Longitudinal progression trajectory of GFR among patients with CKD. Am J Kidney Dis. 2012;59(4):504512. doi:10.1053/j.ajkd.2011.12.009
4. Hannan M, Ansari S, Meza N, et al. Risk factors for CKD progression. Clin J Am Soc Nephrol. 2020:CJN.07830520. doi:10.2215/ CJN.07830520.

5. Chawla LS, Kimmel PL. Acute kidney injury and chronic kidney disease: an integrated clinical syndrome. Kidney Int. 2012;82(5):516524. doi:10.1038/ki.2012.208

6. Doyle JF, Forni LG. Acute kidney injury: short-term and long-term effects. Critical Care. 2016;20(1). doi:10.1186/s13054-016-1353-y

7. Coca SG, Yusuf B, Shlipak MG, Garg AX, Parikh CR. Long-term risk of mortality and other adverse outcomes after acute kidney injury: a systematic review and meta-analysis. Am J Kidney Dis. 2009;53(6):961-973. doi:10.1053/j.ajkd.2008.11.034

8. Yu SM-W, Bonventre JV. Acute kidney injury and progression of diabetic kidney disease. Adv Chronic Kidney Dis. 2018;25(2):166180. doi:10.1053/j.ackd.2017.12.005

9. Patschan D, Müller GA. Acute kidney injury in diabetes mellitus. Int J Nephrol. 2016;2016:1-7. doi:10.1155/2016/6232909

10. Ishani A, Xue JL, Himmelfarb J, et al. Acute kidney injury increases risk of ESRD among elderly. $J$ Am Soc Nephrol. 2009;20(1):223 LP228. doi:10.1681/ASN.2007080837

11. Hoste EAJ, Bagshaw SM, Bellomo R, et al. Epidemiology of acute kidney injury in critically ill patients: the multinational AKI-EPI study. Intensive Care Med. 2015;41(8):1411-1423. doi:10.1007/ s00134-015-3934-7

12. Lysak N, Hashemighouchani H, Davoudi A, et al. Cardiovascular death and progression to end-stage renal disease after major surgery in elderly patients. BJS Open. 2020;4(1):145-156. doi:10.1002/bjs5.50232

13. Huber M, Ozrazgat-Baslanti T, Thottakkara P, Scali S, Bihorac A, Hobson C. Cardiovascular-specific mortality and kidney disease in patients undergoing vascular surgery. JAMA Surg. 2016;151(5):441450. doi:10.1001/jamasurg.2015.4526

14. Girman CJ, Kou TD, Brodovicz K, et al. Risk of acute renal failure in patients with type 2 diabetes mellitus. Diabet Med. 2012;29(5):614621. doi:10.1111/j.1464-5491.2011.03498.x

15. Venot M, Weis L, Clec'h C, et al. Acute kidney injury in severe sepsis and septic shock in patients with and without diabetes mellitus: a multicenter study. PLoS One. 2015;10(5):5. doi:10.1371/journal. pone. 0127411

16. Hapca S, Siddiqui MK, Kwan RSY, et al. The relationship between AKI and CKD in patients with type 2 diabetes: an observational cohort study. J Am Soc Nephrol. 2020;32(1):ASN.2020030323. doi:10.1681/asn.2020030323

17. Levin A, Stevens PE. Summary of KDIGO 2012 CKD guideline: behind the scenes, need for guidance, and a framework for moving forward. Kidney Int. 2014;85(1):49-61. doi:10.1038/ki.2013.444

18. Gross JL, de Azevedo MJ, Silveiro SP, Canani LH, Caramori ML, Zelmanovitz T. Diabetic nephropathy: diagnosis, prevention, and treatment. Diabetes Care. 2005;28(1):164-176. doi:10.2337/ DIACARE.28.1.164

19. Levey AS, Stevens LA, Schmid CH, et al. A new equation to estimate glomerular filtration rate. Ann Intern Med. 2009;150 (9):604-612. doi:10.7326/0003-4819-150-9-200905050-00006

20. Kidney Disease: improving Global Outcomes (KDIGO) Acute Kidney Injury Work Group. KDIGO clinical practice guideline for acute kidney injury. Kidney Int. 2012;2(1):1-138.

21. Singer M, Deutschman CS, Seymour CW, et al. The third international consensus definitions for sepsis and septic shock (sepsis-3). JAMA. 2016;315(8):801-810. doi:10.1001/jama.2016.0287

22. Duan J, Wang C, Liu D, et al. Prevalence and risk factors of chronic kidney disease and diabetic kidney disease in Chinese rural residents: a cross-sectional survey. Sci Rep. 2019;9(1):10408. doi:10.1038/ s41598-019-46857-7

23. Alicic RZ, Rooney MT, Tuttle KR. Diabetic kidney disease: challenges, progress, and possibilities. Clin J Am Soc Nephrol. 2017;12 (12):2032-2045. doi:10.2215/CJN.11491116 
24. Oliveira JFP, Silva CA, Barbieri CD, Oliveira GM, Zanetta DMT, Burdmann EA. Prevalence and risk factors for aminoglycoside nephrotoxicity in intensive care units. Antimicrob Agents Chemother. 2009;53(7):2887 LP- 2891. doi:10.1128/AAC.01430-08

25. Goligorsky MS, Chen J, Brodsky S. Workshop: endothelial cell dysfunction leading to diabetic nephropathy: focus on nitric oxide. Hypertension. 2001;37(2 Pt 2):744-748. doi:10.1161/01. hyp.37.2.744

26. Lenoir O, Milon M, Virsolvy A, et al. Direct action of endothelin-1 on podocytes promotes diabetic glomerulosclerosis. J Am Soc Nephrol. 2014;25(5):1050LP- 1062. doi:10.1681/ASN.2013020195

27. Donate-Correa J, Martín-Núñez E, Muros-de-Fuentes M, MoraFernández C, Navarro-González JF. Inflammatory cytokines in diabetic nephropathy. J Diabetes Res. 2015;2015:948417. doi:10.1155/ 2015/948417

28. Venkatachalam MA, Weinberg JM, Kriz W, Bidani AK. Failed tubule recovery, AKI-CKD transition, and kidney disease progression. $J \mathrm{Am}$ Soc Nephrol. 2015;26(8):1765-1776. doi:10.1681/ASN.2015010006

29. Bagshaw SM, Adhikari NKJ, Burns KEA, et al. Selection and receipt of kidney replacement in critically ill older patients with AKI. Clin J Am Soc Nephrol. 2019;14(4):496-505. doi:10.2215/CJN.05530518

30. Grynberg K, Polkinghorne KR, Ford S, et al. Early serum creatinine accurately predicts acute kidney injury post cardiac surgery. $B M C$ Nephrol. 2017;18(1):1. doi:10.1186/s12882-017-0504-y

31. Thakar CV, Christianson A, Himmelfarb J, Leonard AC. Acute kidney injury episodes and chronic kidney disease risk in diabetes mellitus. Clin J Am Soc Nephrol. 2011;6(11):2567-2572. doi:10.2215/CJN.01120211

32. Monseu M, Gand E, Saulnier P-J, et al. Acute kidney injury predicts major adverse outcomes in diabetes: synergic impact with low glomerular filtration rate and albuminuria. Diabetes Care. 2015;38 (12):2333-2340. doi:10.2337/dc15-1222

33. Sykes L, Asar O, Ritchie J, et al. The influence of multiple episodes of acute kidney injury on survival and progression to end stage kidney disease in patients with chronic kidney disease. PLoS One. 2019;14(7):e0219828. doi:10.1371/journal.pone.0219828

34. James MT, Hemmelgarn BR, Wiebe N, et al. Glomerular filtration rate, proteinuria, and the incidence and consequences of acute kidney injury: a cohort study. Lancet. 2010;376(9758):2096-2103. doi:10.1016/S0140-6736(10)61271-8
35. Waikar SS, Winkelmayer WC. Chronic on acute renal failure: longterm implications of severe acute kidney injury. JAMA. 2009;302 (11):1227-1229. doi:10.1001/jama.2009.1364

36. Okusa MD, Chertow GM, Portilla D. The nexus of acute kidney injury, chronic kidney disease, and world kidney day 2009. Clin J Am Soc Nephrol. 2009;4(3):520-522. doi:10.2215/CJN.06711208

37. Chua H-R, Wong W-K, Ong VH, et al. Extended mortality and chronic kidney disease after septic acute kidney injury. $J$ Intensive Care Med. 2018;35(6):0885066618764617. doi:10.1177/ 0885066618764617

38. He L, Wei Q, Liu J, et al. AKI on CKD: heightened injury, suppressed repair, and the underlying mechanisms. Kidney Int. 2017;92 (5):1071-1083. doi:10.1016/j.kint.2017.06.030

39. Neyra JA, Mescia F, Li X, et al. Impact of acute kidney injury and CKD on adverse outcomes in critically ill septic patients. Kidney Int Rep. 2018;3(6):1344. doi:10.1016/J.EKIR.2018.07.016

40. Muller LMAJ, Gorter KJ, Hak E, et al. Increased risk of common infections in patients with type 1 and type 2 diabetes mellitus. Clin Infect Dis. 2005;41(3):281-288. doi:10.1086/431587

41. Peleg AY, Weerarathna T, McCarthy JS, Davis TME. Common infections in diabetes: pathogenesis, management and relationship to glycaemic control. Diabetes Metab Res Rev. 2007;23(1):3-13. doi:10.1002/dmrr.682

42. Liu J, Xie H, Ye Z, Li F, Wang L. Rates, predictors, and mortality of sepsis-associated acute kidney injury: a systematic review and metaanalysis. BMC Nephrol. 2020;21(1):318. doi:10.1186/s12882-02001974-8

43. Mataloun S, Machado F, Senna A, Guimarães H, Amaral J. Incidence, risk factors and prognostic factors of acute renal failure in patients admitted in an intensive care unit. Crit Care. 2003;7(Suppl 3):P45-P45. doi:10.1186/cc2241

44. Thakar CV, Christianson A, Freyberg R, Almenoff P, Render ML. Incidence and outcomes of acute kidney injury in intensive care units: a veterans administration study. Crit Care Med. 2009;37(9):25522558. doi:10.1097/CCM.0b013e3181a5906f

\section{Publish your work in this journal}

The International Journal of Nephrology and Renovascular Disease is an international, peer-reviewed open-access journal focusing on the pathophysiology of the kidney and vascular supply. Epidemiology, screening, diagnosis, and treatment interventions are covered as well as basic

science, biochemical and immunological studies. The manuscript management system is completely online and includes a very quick and fair peer-review system, which is all easy to use. Visit http://www.dovepress.com/testimonials.php to read real quotes from published authors. 